\title{
A Prospective Study of Early Postoperative Outcome of D2 Gastrectomy: A Tertiary Hospital of Bangladesh
}

\author{
Nabir Hossain*, Ahsan Habib, Quazi Habibullah \\ Department of Surgical Oncology, National Institute of Cancer Research \& Hospital (NICRH), Dhaka, Bangladesh \\ Email address: \\ dnabir7796@gmail.com (N. Hossain) \\ ${ }^{*}$ Corresponding author

\section{To cite this article:} \\ Nabir Hossain, Ahsan Habib, Quazi Habibullah. A Prospective Study of Early Postoperative Outcome of D2 Gastrectomy: A Tertiary \\ Hospital of Bangladesh. Cancer Research Journal. Vol. 9, No. 1, 2021, pp. 53-60. doi: 10.11648/j.crj.20210901.17
}

Received: February 13, 2021; Accepted: February 27, 2021; Published: March 10, 2021

\begin{abstract}
Introduction: Gastrectomy with proper lymphadenectomy is considered the cornerstone of treatment for potentially curable gastric cancer. Stomach cancer is one of the important leading causes of cancer related death in worldwide. Annually, it was diagnosed with 9, 89,600 new cases and 7, 38,000 deaths (10\% of all cancer death) in worldwide. The overall prognosis is not very favorable. However, surgery in the form of gastrectomy is the only treatment modality for a chance of long term survival as well as hope for cure. Aim of the study: Aim of the study was to find outcome of Early Postoperative Outcome of D2 Gastrectomy. Material \& Methods: This prospective study was conducted at the Department of Surgical Oncology, National Institute of Cancer Research \& Hospital (NICRH), Dhaka, Bangladesh from January 2019 to December 2020. A total 80 number of gastric cancer patients who were underwent gastrectomy were considered in this study. Statistical Analysis was performed with help of Epi Info (TM) 7.2.2.2. EPI INFO is a trademark of the Centers for Disease Control and Prevention (CDC). Descriptive statistical analysis was performed to calculate the means with corresponding standard deviations. Results: The mean age (mean \pm SD) of the patients was $49.10 \pm 8.32$ years with range 28-66 years and the median age was 48.5 years. The age group $45-54$ years $(50.0 \%)$ were significantly higher than other age group $(Z=3.26 ; p<0.001)$. Only $5.0 \%$ were with age between $25-34$ years and $27.5 \%$ of the patients were with age $\geq 55$ years. Thus in this study the patients with age between were in higher risk of having gastric cancer. Proportion of males (75.0\%) was significantly higher than that of females $(25.0 \%)(Z=7.07 ; \mathrm{p}<0.0001)$. Conclusion: Surgery for gastric cancer is the only hope for cure or long term survival in the arena of multimodal management of cancers. It provides quick symptom relief by loco regional control, adds to survival of the individual, cure in case of early gastric cancer and proper staging for planning of subsequent adjuvant treatment.
\end{abstract}

Keywords: Early Postoperative, Outcome, D2 Gastrectomy, Lymphadenectomy, Cancer

\section{Introduction}

Gastrectomy with proper lymphadenectomy is considered the cornerstone of treatment for potentially curable gastric cancer [1]. Stomach cancer is one of the important leading causes of cancer related death in worldwide. Annually, it was diagnosed with 9, 89,600 new cases and 7, 38,000 deaths $(10 \%$ of all cancer death) in worldwide. The overall prognosis is not very favorable. However, surgery in the form of gastrectomy is the only treatment modality for a chance of long term survival as well as hope for cure. Thus, prospective study with gastrectomy is necessary for optimum extent of surgery. Surgery in the form of gastric resection was first carried out by Theodre Billroth in
Vienna in 1881. Till now, gastric resections remain standard treatment for carcinoma stomach in the world. Surgical resection offers the only chance of cure in localized Gastric cancer [2]. Radical gastrectomy with D2 lymphadenectomy is now considered as the standard of surgical care in both eastern and western centers [3]. This paradigm shift has brought in newer difficulties in the management of patients who belong to the low socioeconomic group. Combination of adverse features like delayed presentation, significant weight loss, advanced nature of disease increases the risks of surgery several fold. A vast majority of the patients who are treated in the teaching hospital where this study was conducted belong to the low socioeconomic group and present in advanced stages. As experience in laparoscopic gastrectomy for early gastric cancer 
has increased, some surgeons have become concerned about the use of laparoscopic D2 lymphadenectomy in gastric cancer [4]. Additionally, D2 lymphadenectomy is a more challenging procedure than D1 lymphadenectomy [5]. This was mainly due to two factors, one active screening leading to early diagnosis and the other one extensive lymphadenectomy along with gastric resection. As per the Japanese study, if lymph node is not dissected then 5yr overall survival of the patients is about $20.3 \%$. Survival of patients with D1 dissection is $41 \%$ and with D2 dissection is $50-62 \%$. Thus, Japanese literature suggested D2 lymphadenectomy for stomach cancer. It is suggested that high postoperative morbidity and mortality associated with extensive lymph node dissection. In addition, Dutch trial after $15 \mathrm{yr}$ follow up confirmed that D2 gastrectomy has got survival advantages with low cancer specific death rate and low loco regional recurrence compared to D1 gastrectomy. But the question is also remained about high post-operative morbidity and mortality. D2 gastrectomy is also considered to be choice of procedure so far as pathological staging of the disease is concerned. This staging data is very important for planning of adjuvant treatment as cancer management has become multimodal nowadays. So NCCN 2010 has laid down the principle of examining at least 16 lymph nodes for proper pathological staging of stomach cancer. Thus, lighting on modified D2 gastrectomy is necessary for improvement of better survival of gastric patients.

\section{Methodology and Materials}

This prospective study was conducted at the Department of Surgical Oncology, National Institute of Cancer Research \& Hospital (NICRH), Dhaka, Bangladesh from January 2019 to December 2020. As per the inclusion and exclusion criteria a total 80 number of gastric cancer patients who were underwent gastrectomy were considered in this study. Among these cases, biopsy proven and operable cases of adenocarcinoma of stomach enrolled in our institute in the aforementioned period are considered for the study. All the cases of biopsy proven adenocarcinoma of stomach will undergo detailed clinical examination, Chest x-ray, contrast enhanced CT scan of abdomen and pelvis. Only operable and fit patient were planned for surgery. Before surgery diagnostic laparoscopies was carried out and proceed in those cases where there was no gross peritoneal disease. The surgery was carried out as per standard guideline. For distal gastrectomy-1, 3, 4sb, 4d, 5, 6, 7, 8a, 9, 12a irrespective of T stage. For total gastrectomy-1-7, 8a, 9, 11p, 12a (avoiding 11d\&10 group of lymph node as recommended by Japanese cancer association. Resection of pancreatic tail and spleen were avoided until unless they are directly involved by tumors. Lymph node dissections at 10 and 11d were done in proximal cancer. Statistical Analysis was performed with help of Epi Info (TM) 7.2.2.2. EPI INFO is a trademark of the Centers for Disease Control and Prevention (CDC). Descriptive statistical analysis was performed to calculate the means with corresponding standard deviations (SD).

1. Inclusion Criteria

All cases of histologically proven Gastric Adenocarcinoma which were operable as per CT scan abdomen findings.

\section{Exclusion Criteria}

a) Patients with metastatic disease, poor surgical candidates and locally advanced gastric cancer patients requiring neoadjuvant therapy.

b) Patients with peritoneal metastasis on laparoscopic staging.

\section{Results}

The mean age (mean \pm SD) of the patients was $49.10 \pm 8.32$ years with range $28-66$ years and the median age was 48.5 years. The age group $45-54$ years $(50.0 \%)$ were significantly higher than other age group $(Z=3.26 ; p<0.001)$. Only $5.0 \%$ were with age between $25-34$ years and $27.5 \%$ of the patients were with age $\geq 55$ years. Thus in this study the patients with age between were in higher risk of having gastric cancer. Proportion of males $(75.0 \%)$ was significantly higher than that of females $(25.0 \%)$ $(\mathrm{Z}=7.07 ; \mathrm{p}<0.0001)$. The sex ratio was found as Male: Female $=$ 3:1. Thus in this study females were in higher risk of having gastric cancer at a younger age than males. The mean BMI (mean \pm SD) of the patients was $27.10 \pm 2.88 \mathrm{~kg} / \mathrm{m}^{2}$ with range $22.0-34.0 \mathrm{~kg} / \mathrm{m}^{2}$ and the median was $27.0 \mathrm{~kg} / \mathrm{m}^{2}$. Most of the patients were overweight and obese $(75.0 \%)$ followed by normal weight $(25.0 \%)(Z=7.07 ; p<0.001) .20 .0 \%$ of the patients were obese (Table 1). Most of the patients were having dyspepsia $(40.0 \%)$ which was not significantly higher $(Z=1.86 ; \mathrm{p}=0.0629)$ followed by GOO (27.5\%). Only $2.5 \%$ had bleeding and another $2.5 \%$ had loss of appetite. $52.5 \%$ of the patients had no addiction which was higher than addiction $(47.5 \%)$ but it was not significant $(\mathrm{Z}=0.71 ; \mathrm{p}=0.47)$. Out of the all addictions smoking $(27.5 \%)$ was more prevalent than drinking of alcohol (12.5\%) and chewing tobacco $(7.5 \%)$. Most of the patients had no comorbidity $(72.5 \%)$ which was significantly higher than having co-morbidity $(27.5 \%)(\mathrm{Z}=6.36 ; \mathrm{p}<0.0001)$. Out of the all comorbidities diabetes and hypertension (10.0\%) were more prevalent. Only $5.0 \%$ and $2.5 \%$ had diabetes with hypertension and hypothyroidism (2.5\%) respectively (Table 2 ). $35.0 \%$ of the patients had post-operative ileus $>5$ days. $55.0 \%$ of the patients had duration of drainage $>5$ days $(Z=1.41 ; p=0.15)$. Only $10.0 \%$ of the patients had bile leak out of which $7.5 \%$ had bile leak and $2.5 \%$ had mild bile leak. $35.0 \%$ of the patients had postoperative infection. $54(67.5 \%)$ of the patients did not have any other complications (Table 3). Most of the site of tumor was distal $(87.5 \%)$ which was significantly higher than proximal (12.5\%) $(Z=10.60 ; p<0.0001)$. Adenocarcinoma was intestinal $(80.0 \%)$ which was significantly higher than diffuse $(20.0 \%)$ $(p<0.0001)$. Grade of tumor was intermediate $(70.0 \%)$ which was significantly higher than others $(Z=5.65 ; p<0.0001)$. The mean number of lymph node retrieved (mean \pm SD) of the patients was $23.52 \pm 6.95$ with range 13-41 and the median was 22 . The mean number of positive lymph node (mean \pm SD) of the patients was $3.42 \pm 2.80$ with range $0-12$ and the median was 3. All the margin statuses were negative. Only $4(5.0 \%)$ of the cases were having Her-2neu positivity. Test of proportion showed that proportion of Her2 neu positive cases were significantly higher in Proximal $(40.0 \%)$ than that of Distal $(0.0 \%)(Z=7.07 \mathrm{p}<0.0001)$ (Table 4). Increase drainage that is 
more than 5 days is related to low albumin $(<3.2)$ and increase stage of the disease. Similarly ileus is correlated with preoperative $\mathrm{Hb} \%$ level. It is an unknown factor for post-operative ileus. The statistical significance of it is unknown. Regarding other minor complications which are not related to surgical procedure itself there is no significant correlation with hemoglobin, serum albumin, BMI, stage of e disease, age, smoking and the pre-operative co-morbidities. This is improved preoperative management. Only one parameter that is wound infection is significantly higher in our study. LN involvement is one of the strongest prognostic parameters after gastrectomy for survival and recurrence. Precise evaluation of the extent of LN metastasis offers the ability to more accurately predict oncologic outcomes for the individual patient (Tables 5-9).

Table 1. Demographic parameters of patients $(N=80)$.

\begin{tabular}{|c|c|c|c|c|}
\hline Demographic parameters & Number & $\%$ & Test Statistic & p-value \\
\hline \multicolumn{5}{|l|}{ Age Group (in years) } \\
\hline $25-34$ & 4 & 5 & \multirow{4}{*}{$Z=3.26$} & \multirow{4}{*}{$<0.001^{*}$} \\
\hline $35-44$ & 14 & 17.5 & & \\
\hline $45-54$ & 40 & 50 & & \\
\hline$\geq 55$ & 22 & 27.5 & & \\
\hline \multicolumn{5}{|l|}{ Gender (Male: Female $=3: 1$ ) } \\
\hline Male & 60 & 75 & \multirow[t]{3}{*}{$Z=7.07$} & \multirow[t]{2}{*}{$<0.001 *$} \\
\hline Female & 20 & 25 & & \\
\hline \multicolumn{4}{|l|}{$\operatorname{BMI}\left(\mathrm{kg} / \mathrm{m}^{2}\right)$} & \\
\hline 23.0-24.9 (Normal weight) & 20 & 25 & \multirow{3}{*}{$Z=7.07$} & \multirow{3}{*}{$<0.001 *$} \\
\hline 25.0-29.9 (Over weight) & 44 & 55 & & \\
\hline$\geq 30.0$ (Obese) & 16 & 20 & & \\
\hline
\end{tabular}

Table 2. Presenting symptoms, Addiction and Co-morbidities of the patients $(N=80)$.

\begin{tabular}{|c|c|c|c|c|}
\hline Demographic parameters & Number & $\%$ & Test Statistic & p-value \\
\hline & \multicolumn{4}{|c|}{ Presenting symptoms of the patients } \\
\hline Dyspepsia & 32 & & & \\
\hline GOO & 22 & 40 & $Z=1.86$ & $0.0629 \mathrm{NS}$ \\
\hline Malaena & 8 & 27.5 & & \\
\hline Pain & 8 & 10 & & \\
\hline Dysphagia & 6 & 10 & & \\
\hline Bleeding & 2 & 7.5 & & \\
\hline \multirow[t]{2}{*}{ Loss of appetite } & 2 & 2.5 & & \\
\hline & Addiction & & & \\
\hline Smoking & 22 & 27.5 & & \\
\hline Drinking of Alcohol & 10 & 12.5 & & \\
\hline Chewing tobacco & 6 & 7.5 & & \\
\hline \multirow[t]{2}{*}{ No } & 42 & 52.5 & $Z=3.60$ & $<0.001^{*}$ \\
\hline & Co-morbi & & & \\
\hline Diabetes & 8 & 10 & & \\
\hline Hypertension & 8 & 10 & & \\
\hline Diabetes with Hypertension & 4 & 5 & & \\
\hline Hypothyroidism & 2 & 2.5 & & \\
\hline No & 58 & 72.5 & $Z=6.36$ & $<0.001 *$ \\
\hline
\end{tabular}

Table 3. Distribution of different post-operative finding parameters $(N=80)$.

\begin{tabular}{|c|c|c|c|c|}
\hline Demographic parameters & Number & $\%$ & Test Statistic & p-value \\
\hline \multicolumn{5}{|l|}{ Post-operative ileus (in days) } \\
\hline$>5$ & 28 & $35.00 \%$ & & \\
\hline$\leq 5$ & 52 & $65.00 \%$ & $Z=4.24$ & $<0.001 *$ \\
\hline \multicolumn{5}{|l|}{$\begin{array}{l}\text { Duration of drainage } \\
\text { (in days) }\end{array}$} \\
\hline$>5$ & 44 & $55.00 \%$ & $Z=1.41$ & 0.15 \\
\hline$\leq 5$ & 36 & $45.00 \%$ & & \\
\hline \multicolumn{5}{|l|}{ Bile leak and its nature } \\
\hline Bile leak & 6 & $7.50 \%$ & & \\
\hline Mild bile leak & 2 & $2.50 \%$ & & \\
\hline No & 72 & $90.00 \%$ & $Z=11.59$ & $<0.001 *$ \\
\hline \multicolumn{5}{|l|}{ Post-operative Infection } \\
\hline Yes & 28 & $35.00 \%$ & & \\
\hline No & 52 & $65.00 \%$ & $Z=4.24$ & $<0.001^{*}$ \\
\hline \multicolumn{5}{|l|}{ Other complications } \\
\hline Chest infection & 8 & $10.00 \%$ & & \\
\hline Fever & 4 & $5.00 \%$ & & \\
\hline Mild chest infection & 4 & $5.00 \%$ & & \\
\hline
\end{tabular}




\begin{tabular}{lllll}
\hline Demographic parameters & Number & \% & Test Statistic & p-value \\
\hline Pneumothorax & 2 & $2.50 \%$ & & \\
Thrombophlebitis & 8 & $10.00 \%$ & & \\
No & 54 & $67.50 \%$ & $\mathrm{Z}=8.40$ & $<0.001^{*}$ \\
\hline
\end{tabular}

Table 4. Distribution of different histological parameters after surgery $(N=80)$.

\begin{tabular}{|c|c|c|c|c|}
\hline Histological parameters & Number & $\%$ & Test Statistic & p-value \\
\hline \multicolumn{5}{|l|}{ Site of tumour } \\
\hline Distal & 70 & 87.5 & $Z=10.60$ & $<0.001 *$ \\
\hline Proximal & 10 & 12.5 & & \\
\hline \multicolumn{5}{|l|}{ Type of } \\
\hline \multicolumn{5}{|l|}{ Adenocarcinoma } \\
\hline Diffuse & 16 & $20.00 \%$ & $Z=8.48$ & $<0.001 *$ \\
\hline Intestinal & 64 & $80.00 \%$ & & \\
\hline \multicolumn{5}{|l|}{ Grade of Tumour } \\
\hline High & 22 & $27.50 \%$ & & \\
\hline Intermediate & 56 & $70.00 \%$ & $Z=5.65$ & $<0.001 *$ \\
\hline Low & 2 & $2.50 \%$ & & \\
\hline \multicolumn{5}{|l|}{ Pathological stage } \\
\hline IA & 2 & $2.50 \%$ & & \\
\hline IB & 6 & $7.50 \%$ & & \\
\hline IIA & 12 & $15.00 \%$ & & \\
\hline IIB & 26 & $32.50 \%$ & $Z=2.98$ & 0.0028 \\
\hline IIIA & 12 & $15.00 \%$ & & \\
\hline IIIB & 12 & $15.00 \%$ & & \\
\hline IIIC & 10 & $12.50 \%$ & & \\
\hline \multicolumn{5}{|l|}{ Status of LVI/PNI } \\
\hline LVI and PNI+ & 16 & $20.00 \%$ & & \\
\hline LVI+ & 48 & $60.00 \%$ & $Z=9.19$ & $<0.001 *$ \\
\hline PNI+ & 2 & $2.50 \%$ & & \\
\hline Negative & 14 & $17.50 \%$ & & \\
\hline \multicolumn{5}{|l|}{ Her-2neu Status } \\
\hline Negative & 76 & $95.00 \%$ & $Z=12.72$ & $<0.001 *$ \\
\hline Positive & 4 & $5.00 \%$ & & \\
\hline
\end{tabular}

Table 5. Comparison of duration of drainage with different parameters $(N=80)$.

\begin{tabular}{|c|c|c|c|c|c|}
\hline \multirow{2}{*}{ Parameters } & \multirow{2}{*}{$\begin{array}{l}\text { Duration of drainage }>5 \text { days } \\
(n=44)\end{array}$} & \multirow{2}{*}{$\begin{array}{l}\text { Duration of drainage } \leq 5 \text { days } \\
(n=36)\end{array}$} & \multirow{2}{*}{ Test- statistic } & \multirow{2}{*}{ p-value } & \multirow{2}{*}{$\begin{array}{l}\text { Odds Ratio with 95\% } \\
\text { Confidence Interval }\end{array}$} \\
\hline & & & & & \\
\hline $\begin{array}{l}\text { Serum albumin } \\
(\text { Mean } \pm \text { SD) }\end{array}$ & $3.18 \pm 0.58$ & $3.70 \pm 0.60$ & $\mathrm{t}_{38}=2.77$ & $0.0086^{*}$ & NA \\
\hline $\begin{array}{l}\text { Level of Pre-op } \\
\mathrm{Hb}(\text { Mean } \pm \mathrm{SD})\end{array}$ & $10.50 \pm 1.92$ & $12.00 \pm 2.53$ & $\mathrm{t}_{38}=2.13$ & $0.0397 *$ & NA \\
\hline $\begin{array}{l}\text { BMI }\left(\mathrm{kg} / \mathrm{m}^{2}\right) \\
(\mathrm{Mean} \pm \mathrm{SD})\end{array}$ & $26.68 \pm 3.46$ & $27.61 \pm 1.94$ & $\mathrm{t}_{38}=1.01$ & 0.31 & NA \\
\hline $\begin{array}{l}\text { Age (years) (Mean } \\
\pm \mathrm{SD})\end{array}$ & $48.36 \pm 9.67$ & $50.00 \pm 6.48$ & $\mathrm{t}_{38}=0.61$ & 0.54 & NA \\
\hline \multicolumn{6}{|l|}{ Smoking } \\
\hline Yes & $10(22.7 \%)$ & $612(33.3 \%)$ & \multirow{2}{*}{$\mathrm{c}^{2}=0.55$} & \multirow{2}{*}{0.45} & \multirow{3}{*}{ [OR-0.58 $(0.14,2.38]$} \\
\hline No & $34(77.3 \%)$ & $24(66.7 \%)$ & & & \\
\hline \multicolumn{5}{|l|}{ Stage of the disease } & \\
\hline IA & $0(0.0 \%)$ & $2(5.6 \%)$ & \multirow{7}{*}{$c^{2}=16.12$} & \multirow{7}{*}{$0.013^{*}$} & \multirow{7}{*}{ NA } \\
\hline IB & $2(4.5 \%)$ & $4(11.1 \%)$ & & & \\
\hline IIA & $2(4.5 \%)$ & $10(27.8 \%)$ & & & \\
\hline IIB & $10(22.7 \%)$ & $16(44.4 \%)$ & & & \\
\hline IIIA & $8(18.2 \%)$ & $4(11.1 \%)$ & & & \\
\hline IIIB & $12(27.3 \%)$ & $0(0.0 \%)$ & & & \\
\hline IIIC & $10(22.7 \%)$ & $0(0.0 \%)$ & & & \\
\hline \multicolumn{6}{|l|}{ Presence of diabetes } \\
\hline Yes & $8(18.2 \%)$ & $4(11.1 \%)$ & \multirow{2}{*}{$\mathrm{c}^{2}=0.38$} & \multirow{2}{*}{0.53} & \multirow{2}{*}{ [OR-1.77 $(0.28,11.03]$} \\
\hline No & $36(81.8 \%)$ & $32(88.9 \%)$ & & & \\
\hline \multicolumn{6}{|l|}{ Site of the disease } \\
\hline Distal & $34(77.3 \%)$ & $36(100.0 \%)$ & & \multirow{2}{*}{$0.04 *$} & \\
\hline Proximal & $10(22.7 \%)$ & $0(0.0 \%)$ & Fisher Exact Test & & \\
\hline
\end{tabular}


Table 6. Comparison of duration of Ileus with different parameters $(N=80)$.

\begin{tabular}{|c|c|c|c|c|c|}
\hline \multirow{2}{*}{ Parameters } & Duration of & Duration of & \multirow{2}{*}{$\begin{array}{l}\text { Test- } \\
\text { statistic }\end{array}$} & \multirow{2}{*}{ p-value } & \multirow{2}{*}{$\begin{array}{l}\text { Odds Ratio with } 95 \% \\
\text { Confidence Interval }\end{array}$} \\
\hline & Ileus $>5$ days $(n=28)$ & Ileus $\leq 5$ days $(n=52)$ & & & \\
\hline $\begin{array}{l}\text { Serum albumin } \\
\text { (Mean } \pm \mathrm{SD} \text { ) }\end{array}$ & $3.10 \pm 0.59$ & $3.58 \pm 0.60$ & $\mathrm{t} 38=1.30$ & 0.2 & NA \\
\hline $\begin{array}{l}\text { Level of Pre-op Hb } \\
\text { (Mean } \pm \text { SD) }\end{array}$ & $10.07 \pm 1.71$ & $5.81 \pm 2.41$ & $\mathrm{t} 38=5.85$ & $<0.00018^{*}$ & NA \\
\hline $\begin{array}{l}\text { BMI }\left(\mathrm{kg} / \mathrm{m}^{2}\right) \\
(\text { Mean } \pm \mathrm{SD})\end{array}$ & $26.71 \pm 3.53$ & $27.30 \pm 2.52$ & $\mathrm{t} 38=0.61$ & 0.54 & NA \\
\hline $\begin{array}{l}\text { Age (years) } \\
(\text { Mean } \pm \mathrm{SD})\end{array}$ & $50.00 \pm 10.42$ & $48.61 \pm 7.14$ & $\mathrm{t} 38=0.49$ & 0.62 & NA \\
\hline \multicolumn{6}{|l|}{ Smoking } \\
\hline Yes & $4(14.3 \%)$ & $18(34.6 \%)$ & \multirow[t]{2}{*}{$\chi^{2}$} & \multirow[t]{2}{*}{0.16} & \multirow[t]{2}{*}{ [OR-0.31 $(0.05,1.72]$} \\
\hline No & $24(85.7 \%)$ & $34(65.4 \%)$ & & & \\
\hline \multicolumn{6}{|l|}{ Stage of the disease } \\
\hline IA & $0(0.0 \%)$ & $2(3.8 \%)$ & \multirow{7}{*}{$\chi^{2}=12.86$} & \multirow{7}{*}{$0.045^{*}$} & \multirow{7}{*}{ NA } \\
\hline IB & $0(0.0 \%)$ & $6(11.5 \%)$ & & & \\
\hline IIA & $0(0.0 \%)$ & $12(23.1 \%)$ & & & \\
\hline IIB & $6(21.4 \%)$ & $20(38.5 \%)$ & & & \\
\hline IIIA & $8(28.6 \%)$ & $4(7.7 \%)$ & & & \\
\hline IIIB & $8(28.6 \%)$ & $4(7.7 \%)$ & & & \\
\hline IIIC & $6(21.4 \%$ & $4(7.7 \%)$ & & & \\
\hline \multicolumn{6}{|l|}{ Presence of diabetes } \\
\hline Yes & $4(14.3 \%)$ & $8(15.4 \%)$ & \multirow{2}{*}{$\chi^{2}=0.13$} & \multirow{2}{*}{0.71} & \multirow{2}{*}{ [OR-0.91 $(0.14,5.75]$} \\
\hline No & $24(85.7 \%)$ & $44(84.6 \%)$ & & & \\
\hline \multicolumn{6}{|l|}{ Site of the disease } \\
\hline Distal & $24(85.7 \%)$ & $46(88.5 \%)$ & \multirow{2}{*}{$\chi^{2}=0.06$} & \multirow{2}{*}{0.8} & \multirow{2}{*}{ [OR-0.78 $(0.11,5.34]$} \\
\hline Proximal & $4(14.3 \%)$ & $6(11.5 \%)$ & & & \\
\hline
\end{tabular}

Table 7. Comparison of Wound Infection with different parameters $(N=80)$.

\begin{tabular}{|c|c|c|c|c|c|}
\hline \multirow{2}{*}{ Parameters } & Wound Infection & No Wound Infection & \multirow{2}{*}{ Test-statistic } & \multirow{2}{*}{ p-value } & \multirow{2}{*}{$\begin{array}{l}\text { Odds Ratio with } 95 \% \\
\text { Confidence Interval }\end{array}$} \\
\hline & $(n=52)$ & $(n=28)$ & & & \\
\hline $\begin{array}{l}\text { Serum albumin } \\
\text { (Mean } \pm \text { SD) }\end{array}$ & $3.25 \pm 0.54$ & $3.50 \pm 0.68$ & $\mathrm{t}_{38}=1.27$ & 0.21 & NA \\
\hline $\begin{array}{l}\text { Level of Pre-op Hb } \\
(\text { Mean } \pm \mathrm{SD})\end{array}$ & $10.28 \pm 2.44$ & $11.65 \pm 2.13$ & $\mathrm{t}_{38}=1.76$ & 0.0865 & NA \\
\hline $\begin{array}{l}\text { BMI }\left(\mathrm{kg} / \mathrm{m}^{2}\right) \\
(\text { Mean } \pm \mathrm{SD})\end{array}$ & $27.57 \pm 3.58$ & $26.48 \pm 2.47$ & $\mathrm{t}_{38}=1.01$ & 0.31 & NA \\
\hline $\begin{array}{l}\text { Age (years) } \\
\text { (Mean } \pm \text { SD) }\end{array}$ & $49.42 \pm 8.89$ & $48.92 \pm 8.18$ & $\mathrm{t}_{38}=0.17$ & 0.86 & NA \\
\hline \multicolumn{6}{|l|}{ Smoking } \\
\hline Yes & $10(35.7 \%)$ & $12(23.1 \%)$ & \multirow{2}{*}{$c^{2}=0.72$} & \multirow{2}{*}{0.39} & \multirow{2}{*}{ [OR-1.851 $(0.44,7.69]$} \\
\hline No & $18(64.3 \%)$ & $40(76.9 \%)$ & & & \\
\hline \multicolumn{6}{|l|}{ Stage of the disease } \\
\hline IA & $0(0.0 \%)$ & $2(3.8 \%)$ & \multirow{7}{*}{$c^{2}=7.33$} & \multirow{7}{*}{0.29} & \multirow{7}{*}{ NA } \\
\hline IB & $0(0.0 \%)$ & $6(11.5 \%)$ & & & \\
\hline IIA & $2(7.1 \%)$ & $10(11.5 \%)$ & & & \\
\hline IIB & $14(50.0 \%)$ & $12(23.1 \%)$ & & & \\
\hline IIIA & $2(7.1 \%)$ & $10(19.2 \%)$ & & & \\
\hline IIIB & $4(14.3 \%)$ & $8(15.4 \%)$ & & & \\
\hline IIIC & $6(21.4 \%)$ & $2(7.7 \%)$ & & & \\
\hline \multicolumn{6}{|l|}{ Presence of diabetes } \\
\hline Yes & $6(21.4 \%)$ & $6(11.5 \%)$ & \multirow{2}{*}{$c^{2}=0.69$} & \multirow{2}{*}{0.4} & \multirow{2}{*}{ [OR-2.09 $(0.36,12.08]$} \\
\hline No & $22(78.6 \%)$ & $46(88.5 \%)$ & & & \\
\hline \multicolumn{6}{|l|}{ Site of the disease } \\
\hline Distal & $22(78.6 \%)$ & $48(92.3 \%)$ & \multirow[t]{2}{*}{$\mathrm{c}^{2}=1.56$} & \multirow{2}{*}{0.21} & \multirow{2}{*}{ [OR-0.30 $(0.44,2.09]$} \\
\hline Proximal & $6(21.4 \%)$ & $4(7.7 \%)$ & & & \\
\hline
\end{tabular}


Table 8. Comparison of Chest Infection with different parameters $(N=80)$.

\begin{tabular}{|c|c|c|c|c|c|}
\hline \multirow{2}{*}{ Parameters } & Chest Infection & No Chest Infection & \multirow{2}{*}{ Test-statistic } & \multirow{2}{*}{ p-value } & \multirow{2}{*}{$\begin{array}{l}\text { Odds Ratio with } 95 \% \\
\text { Confidence Interval }\end{array}$} \\
\hline & $(n=12)$ & $(n=68)$ & & & \\
\hline $\begin{array}{l}\text { Serum albumin } \\
\text { (Mean } \pm \text { SD) }\end{array}$ & $3.18 \pm 0.41$ & $3.54 \pm 0.66$ & $\mathrm{t}_{38}=1.28$ & 0.2 & NA \\
\hline $\begin{array}{l}\text { Level of Pre-op Hb } \\
\text { (Mean } \pm \text { SD) }\end{array}$ & $11.21 \pm 1.63$ & $11.16 \pm 2.43$ & $\mathrm{t}_{38}=0.05$ & 0.96 & NA \\
\hline $\begin{array}{l}\mathrm{BMI}\left(\mathrm{kg} / \mathrm{m}^{2}\right) \\
(\mathrm{Mean} \pm \mathrm{SD})\end{array}$ & $26.33 \pm 3.14$ & $27.23 \pm 2.87$ & $\mathrm{t}_{38}=0.69$ & 0.49 & NA \\
\hline $\begin{array}{l}\text { Age (years) } \\
\text { (Mean } \pm \text { SD) }\end{array}$ & $51.00 \pm 13.71$ & $48.76 \pm 7.26$ & & & NA \\
\hline \multicolumn{6}{|l|}{ Smoking } \\
\hline $\begin{array}{l}\text { Yes } \\
\text { No }\end{array}$ & $\begin{array}{l}6(50.0 \%) \\
6(50.0 \%)\end{array}$ & $\begin{array}{l}18(23.5 \%) \\
52(76.5 \%)\end{array}$ & $c^{2}=1.79$ & 0.18 & [OR-3.25 $(0.54,19.38]$ \\
\hline \multicolumn{6}{|l|}{ Stage of the disease } \\
\hline IA & $0(0.0 \%)$ & $2(2.9 \%)$ & \multirow{7}{*}{$c^{2}=6.35$} & \multirow{7}{*}{0.38} & \multirow{7}{*}{ NA } \\
\hline IB & $0(0.0 \%)$ & $6(8.8 \%)$ & & & \\
\hline IIA & $0(0.0 \%)$ & $12(17.6 \%)$ & & & \\
\hline IIB & $2(16.7 \%)$ & $24(35.3 \%)$ & & & \\
\hline IIIA & $2(16.7 \%)$ & $10(14.7 \%)$ & & & \\
\hline IIIB & $4(33.3 \%)$ & $8(11.8 \%)$ & & & \\
\hline IIIC & $4(33.3 \%)$ & $6(8.8 \%)$ & & & \\
\hline \multicolumn{6}{|l|}{ Presence of diabetes } \\
\hline Yes & $4(33.3 \%)$ & $8(11.8 \%)$ & \multirow{3}{*}{$\mathrm{c}^{2}=1.86$} & \multirow{2}{*}{0.17} & \multirow{2}{*}{ [OR-3.75 $(0.51,27.49]$} \\
\hline No & $8(66.7 \%)$ & $60(88.2 \%)$ & & & \\
\hline \multicolumn{5}{|l|}{ Site of the disease } & \\
\hline Distal & $8(66.7 \%)$ & $62(91.2 \%)$ & \multirow{2}{*}{$\mathrm{c}^{2}=2.80$} & \multirow{2}{*}{0.09} & \multirow{2}{*}{ [OR-0.19 $(0.02,1.53]$} \\
\hline Proximal & $4(33.3 \%)$ & $6(8.8 \%)$ & & & \\
\hline
\end{tabular}

Table 9. Comparison of Lymphnodes retrieved by surgery $(N=80)$ 。

\begin{tabular}{llll}
\hline \multirow{2}{*}{ Parameters } & Correlation & \multirow{2}{*}{ Test Statistic } & p-value \\
\cline { 2 - 2 } & $\mathbf{( r )}$ & & 0.0695 \\
Correlation with age & $\mathrm{r}=-0.29$ & & $0.0070^{*}$ \\
Correlation with BMI & $\mathrm{r}=-0.42$ & & $<0.0001^{*}$ \\
Correlation with Stage of disease & $\mathrm{r}=0.75$ & $\mathrm{t}$-test & \\
Stage of the disease & Lymphnodes retrieved & $\mathrm{t}_{38}=6.38$ & $<0.0001^{*}$ \\
Stage-I $(\mathrm{n}=8)$ & $15.75 \pm 2.50$ & & \\
Stage-II $(\mathrm{n}=38)$ & $19.73 \pm 2.82$ & & $<0.0001^{*}$ \\
Stage-III $(\mathrm{n}=34)$ & $29.58 \pm 6.03$ & $\mathrm{t}_{38}=4.48$ & \\
Site of the disease & $22.00 \pm 5.79$ & \\
Distal $(\mathrm{n}=70)$ & $34.20 \pm 4.76$ & & \\
Proximal $(\mathrm{n}=10)$ & & & \\
\hline
\end{tabular}

\section{Discussion}

The mean age group was 49.10 , with lowest one is 28 and highest one is 66 . The most common age group affected was 45-54. This was quite different from observation made by Degiuli et al [6] and H Danielson et al [7] who have majority of patients in the age group of more than $60 \mathrm{yrs}$. The proportion of females is considerably low in comparison to males. The male: female ratio is $3: 1$ which is very much same as that by W. G Lewis et al [8] and P. Edwards et al [9]. So far BMI is concerned hardly any patient is underweight. Majority have a BMI above 25. This indicates significant weight loss in ca stomach is an accompaniment of advanced or inoperable disease rather than socioeconomic status as majority of the patients are of low socioeconomic status. That means significant weight loss is probably an indirect sign of inoperability. Among the symptoms the most common are in the order of frequency are dyspepsia, GOO, malaena or bleeding, pain abdomen, anorexia. It is same as the observation made by Wanabe $\mathrm{H} \mathrm{J}$ et al [10]. None of the patients have any surgical procedure or any major hospitalization. In fact we have excluded those patients having previous abdominal surgery so that the outcome is not to be affected by other factors. The most common site of the tumour was distal about in $87.5 \%$ of the cases. This was almost like that of Huang et al. (80\%) [11]. So it is implied that the complication rate increases with increase in stage of disease. The complication in the form of wound infection was present in $35 \%$ of cases. This is probably higher in comparison to any standard data. A Nafae et al [12] presented in his paper $13 \%$ of wound infection D1 and $3.7 \%$ wound infection in D2 group. The increased rate of wound infection is a sign of poor maintenance of infection control protocol 
preoperatively as well as some inferior quality of drugs being prescribed to patients. The majority of patients have low or intermediate grade tumour and majority of intestinal variety. Tumoursin our study were differentiated carcinomas. This finding was contradictory to Chang Ming Huang et al [11] (differentiated growths $=22.1 \%$ and undifferentiated $=77.9 \%$ ) and Asada Methasate et al [13] (differentiated $=40 \%$ and undifferentiated $=60 \%$ ) So far early post-op course is concerned we have considered bile leak, increased drainage for more than 5 days, prolonged ileus, wound infection as more specific complications related to surgery itself. Whereas other nonspecific complications as chest infection, fever, bedsores, thrombophlebitis incidence was $32.5 \%$ of cases. The above complications lead to enhanced (slightly) hospital stay and ICU stay. But none contributed to mortality. This is in sharp contrast to the earlier studies which have reported mortality in D2 gastrectomy. Mortality rates associated with radical resection of stomach cancer have improved greatly owing to more rigorous patient selection and development in the surgical techniques and postoperative care $[14,15]$. LN involvement is one of the strongest prognostic parameters after gastrectomy for survival and recurrence. In a Japanese classification, the extent of LN dissection was represented by D0-D3 using the LN station system. The system is complicated and is hard to use due to variations in each category [16]. HER2 over expression was more commonly seen in the intestinal-type than diffuse-type cancers (32\% versus 6\%) [17]. There was also a high concordance rate noted between IHC and FISH HER2 results from primary and metastatic sites. Very interestingly we have found that in around $40 \%$ of proximal cases the Her 2 neu is positive and mostly in diffuse variety of the tumours. This is almost contrary to the data presented. So as a generalized guideline doing Her2neu testing in all cases of cancer stomach cannot be recommended in Indian scenario. But as a part of individualized medicine it should be done in metastatic gastric cancer considering financial and physiological status of the patients.

\section{Limitations of the Study}

The study had a too small sample size. The study was conducted in a single center which doesn't reflect the original scenario of Bangladesh. So here need a large multi scale, multi center countrywide study for authentic outcome.

\section{Conclusion and Recommendations}

Surgery for gastric cancer is the only hope for cure or long term survival in the arena of multimodal management of cancers. It provides quick symptom relief by loco regional control, adds to survival of the individual, cure in case of early gastric cancer and proper staging for planning of subsequent adjuvant treatment. However controversy regarding the extent of surgery still remains today after so much advance in the field of cancer. This modification improves postoperative outcome significantly. With proper patient selection, proper postop care and improvement in surgical techniques the mortality (procedure specific) can be zero almost.

\section{Conflict of Interest}

All the authors do not have any possible conflicts of interest.

\section{References}

[1] Lin J-X, Huang C-M, Zheng C-H, Li P, Xie J-W, Wang J-B, et al. Surgical outcomes of 2041 consecutive laparoscopic gastrectomy procedures for gastric cancer: a large-scale case control study. PLoS One. 2015; 10: e0114948.

[2] Badgwell B, Blum M, Estrella J, Chiang YJ, Das P, Matamoros A, et al. Predictors of survival in patients with resectable gastric cancer treated with preoperative chemo radiation therapy and gastrectomy. J Am Coll Surgeons. 2015; 221 (1): 83-90.

[3] Degiuli M, De Manzoni G, Di Leo A, D’Ugo D, Galasso E, Marrelli D, et al. Gastric cancer: Current status of lymph node dissection. World J Gastroenterol. 2016; 22 (10): 2875.

[4] Chen Q-Y, Huang C-M, Lin J-X, Zheng C-H, Li P, Xie J-W, et al. Laparoscopy-assisted versus open D2 radical gastrectomy for advanced gastric cancer without serosal invasion: a case control study. World J Surg Oncol. 2012; 10: 248.

[5] Park YK, Yoon HM, Kim YW, Park JY, Ryu KW, Lee YJ, et al. Laparoscopy assisted versus Open D2 distal gastrectomy for advanced gastric cancer: results from a randomized phase II multicenter clinical trial (COACT 1001). Ann Surg. 2018; 267: 638-45.

[6] Degiuli, M., Sasasko, M., Ponti, A., et al. (2014) Morbidity and Mortality in Italian Gastric Cancer Study Group Randomized Clinical Trial of D1 versus Modified D2 Resection for Gastric Cancer. British Journal of Surgery, 101, 23-31.

[7] Danielson, H., Kokkola, A., Kiviluoto, T., Siren, J., et al. (2007) Clinical Outcome after D1 versus D2-3 Gastrectomy for Treatment of Gastric Cancer. Scandinavian Journal of Surgery, 96, 35-40.

[8] Lewis, W. G., Edwards, P., et al. (2002) D2 or Not D2? The Gastrectomy Question. Gastric Cancer, 5, 29-34.

[9] Edwards, P., Blackshaw, G. R. J. C., Lewis, W. G., Barry, J. D., Allison, M. C. and Jones, D. R. B. (2004) Prospective Comparison of D1 vs. Modified D2 Gastrectomy for Carcinoma. British Journal of Cancer, 90, 1888-1892.

[10] Wanebo, H. J., Kennedy, B. J., Chmiel, J., Steele, G., Winchester, D. and Osteen, R. (1993) Cancer of Stomach. A Patient Care Study by American College of Surgeons. Annals of Surgery, 218, 583-592.

[11] Huang, C.-M., Lin, J.-X., Zheng, C.-H., Li, P., Xie, J.-W. And Wang, J.-B. (2011) Impact of the Number of Dissected Lymph Nodes on Survival for Gastric Cancer after Distal Subtotal Gastrectomy. Gastroenterology Research and Practice, 2011, Article ID: 476014. 
[12] Alfar Nafae, Raiees Ahmad, Amber Aliya, Yawar Nisar, Pervaze Salam, Imtiyaz Ahmad Surgical Science, 2016, 7, 1326.

[13] Methasate, A., Trakarnsanga, A., Akaraviputh, T., Chinsawangwathanakol, V. and Lohsiriwat, D. (2010) Lymph Node Metastasis in Gastric Cancer: Result of Modified D2 Dissection. Journal of the Medical Association of Thailand, 93, 310-317.

[14] Sano, T., Sasako, M., Yamamoto, S., Nashimoto, A., Kurita, A., Hiratsuka, M., et al. (2004) Gastric Cancer Surgery: Morbidity and Mortality Results from a Prospective Randomized Controlled Trial Comparing Modified D2 and Extended Para-Aortic Lymphadenectomy_Japan Clinical Oncology Group Study 9501. Journal of Clinical Oncology, $22,2767-2773$.
[15] Kinoshita, T., Maruyama, K., Sasako, M. and Okajima, K. (1993) Treatment Results of Gastric Cancer Patients: Japanese Experience. In: Nishi, M., Ichikawa, H., Nakajima, T., Maruyama, K. and Tahara, E., Eds., Gastric Cancer, Springer, Tokyo, 319- 330.

[16] Song Y., Huang J., Wang J. (2010) [Relationship between HER2/neu gene amplification and protein expression and prognosis in patients with advanced gastric carcinoma]. Chin $\mathrm{J}$ Cancer 29: 76-81.

[17] Bang Y., Chung H., Xu J., Lordick F., Sawaki A., Lipatov O., Al-Sakaff N., et al. (2009) Pathological features of advanced gastric cancer (GC): Relationship to human epidermal growth factor receptor 2 (HER2) positivity in the global screening programme of the ToGA trial. J Clin Oncol 27 (215 Suppl.): abstract 4556. 